Somnologie $2016 \cdot 20: 239-240$

DOI 10.1007/s11818-016-0094-0

C) Springer Medizin Verlag Berlin 2016

CrossMark

\author{
Richard Schulz $\cdot$ Holger Hein ${ }^{2,3} \cdot$ Martina Siedler $^{4}$ \\ 'Pneumologie, Schlaf- und Beatmungsmedizin, HELIOS Klinik Bad Schwalbach, Bad Schwalbach, \\ Deutschland \\ ${ }^{2}$ Schlaflabor, St. Adolf-Stift, Reinbek, Deutschland \\ ${ }^{3}$ Praxis für Innere Medizin, Pneumologie, Schlafmedizin, Reinbek, Deutschland \\ ${ }^{4}$ Springer Medizin Verlag, Heidelberg, Deutschland
}

\title{
Zertifizierte Fortbildung in der Zeitschrift Somnologie
}

Sehr geehrte, liebe Leserinnen und Leser,

wir freuen uns sehr, dass mit dieser Ausgabe der Somnologie die Rubrik „CME Zertifizierte Fortbildung " gestartet wird.

"Continuing medical education" (CME) ist uns als Sektionsherausgebern ein besonderes Anliegen. Denn nicht nur neueste Forschungsergebnisse sollten im Fokus einer medizinischen Fachgesellschaft wie der DGSM (Deutsche Gesellschaft für Schlafforschung und Schlafmedizin) stehen, sondern auch die Vermittlung des derzeitigen Kenntnisstandes, z. B. zu bestimmten Krankheitsbildern oder diagnostischen und therapeutischen Verfahren. Dies kann im Rahmen der Somnologie mehr oder weniger nur komprimiert erfolgen, die Autoren garantieren als jeweilige Experten der behandelten Thematik jedoch eine entsprechende Aktualität ihrer Beiträge.

Die CME-Arbeiten, die von nun an in jedem Heft der Somnologie erscheinen werden, sollen dazu dienen, vorhandenes schlafmedizinisches Wissen aufzufrischen und zu vertiefen, aber auch Wissensdefizite zu beheben, die bei jedem von uns bestehen. Letzteres trifft wohl in besonderem Maße für das vergleichsweise junge und interdisziplinäre Fach der Schlafmedizin zu. Welcher Psychiater oder Neurologe kennt schon im Detail die pathophysiologischen Grundlagen der schlafbezogenen Atmungsstörungen und welcher Pneumologe die Pharmakotherapie der Narkolepsie? Insofern hoffen wir, dass mit der neuen CME-Rubrik in der Somnologie eine Lücke im Sinne einer umfassenden schlafmedizinischen Fort- und Weiterbildung geschlossen wird. Hierzu gehört natürlich auch, dass die Inhalte der CME-Artikel sukzessive die Pflichtgebiete bei der Ausbildung zum Somnologen abdecken werden.

Der erste CME-Beitrag in der Somnologie wurde dankenswerterweise von Herrn Prof. Thomas Wetter aus Regensburg gestaltet. Thema ist das Restlesslegs-Syndrom (RLS), zu dem es in den letzten Jahren erhebliche Erkenntnisgewinne, z. B. bezüglich Pathophysiologie und medikamentöser Therapie, gegeben hat. Auch wird zunehmend deutlich, dass das RLS ähnlich wie andere Schlafstörungen, wie z. B. die obstruktive Schlafapnoe oder die chronische Insomnie, das kardiovaskuläre Risiko erhöhen kann. Hervorzuheben ist schließlich, dass die Diagnose des RLS unverändert auf der Erhebung einer gezielten, symptomorientierten Anamnese basiert, wozu unserer Meinung nach jeder schlafmedizinisch Tätige in der Lage sein sollte.

Zum praktischen Ablauf der zertifizierten Fortbildung in der Zeitschrift Somnologie ist Folgendes zu sagen: Wie Sie wissen, ist seit 2004 bzw. 2005 der Nachweis ärztlicher Fortbildung für alle Ärzte, ob niedergelassen oder im Krankenhaus tätig, zur gesetzlich verankerten Pflicht geworden. SpringerMedizin.de bietet Ärzten seit Beginn der Fortbildungspflicht mit seinen deutschsprachigen medizinischen Fachzeitschriften die Möglichkeit, online CME-Punkte zu sammeln.

Über das CME-Fortbildungsangebot von SpringerMedizin.de: 
- zertifiziert von der Ärztekammer Nordrhein und damit bundesweit anerkennungsfähig,

- CME-Teilnahme ausschließlich über das Internet,

- CME-Fortbildungen sind 12 Monate aktiv,

- pro CME-Fortbildung ein CMEFragebogen mit 10 Prüfungsfragen,

- 3 Fortbildungspunkte bei mindestens 70 \% richtig beantworteten Fragen,

- Ergebnis wird online sofort mitgeteilt,

- automatische Punkteübertragung bei Angabe der EFN (Einheitliche Fortbildungsnummer),

- Bescheinigung kann als PDF-Datei gespeichert werden.

Abonnenten von Somnologie können das CME-Angebot ihrer Zeitschrift unter www.springermedizin.de/cme kostenlos nutzen.

Die Rubrikherausgeber und die Redaktion von Somnologie wünschen Ih- nen, den Leserinnen und Lesern, eine interessante Lektüre und natürlich viel Erfolg bei der Beantwortung der Fragen.

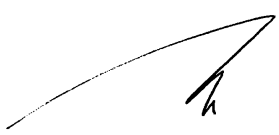

Prof. Dr. med. Richard Schulz, MHBA Bad Schwalbach

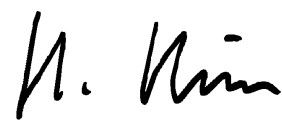

Dr. med. Holger Hein Reinbek

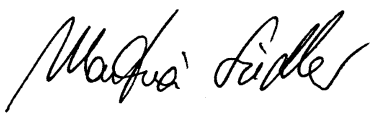

Martina Siedler

Head of Educational Publishing, Springer Medizin

\section{Korrespondenzadresse}

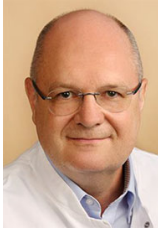

Prof. Dr. med. R. Schulz MHBA

Pneumologie, Schlaf- und Beatmungsmedizin, HELIOS Klinik Bad Schwalbach Emser Straße 29-31, 65307 Bad Schwalbach, Deutschland richard.schulz@helioskliniken.de

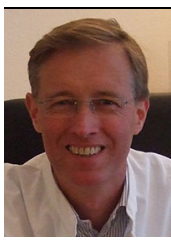

\section{Dr. med. H. Hein} Schlaflabor, St. Adolf-Stift Hamburger Str. 41, 21465 Reinbek, Deutschland hh@dr-holger-hein.de

Interessenkonflikt. R. Schulz, H. Hein und M. Siedler geben an, dass kein Interessenkonflikt besteht.

\section{CME-Punkte sammeln in 3 Schritten}

Als Zeitschriftenabonnent stehen Ihnen unter CME.SpringerMedizin.de alle zertifizierten Fortbildungskurse Ihrer Zeitschrift zur Verfügung.

So einfach sammeln Sie CME-Punkte:

Registrieren

Um CME-Fortbildungen bearbeiten zu können, müssen Sie sich einmalig unter www.springermedizin.de/register registrieren. Bitte geben Sie bei der Registrierung die Lieferadresse Ihrer abonnierten Zeitschrift an, damit die Angaben Ihres OnlineAccounts mit den Angaben Ihres Zeitschriften-Abonnements übereinstimmen. Die CME-Beiträge werden anschließend automatisch freigeschaltet.

Beitrag auswählen

Sobald Sie sich mit Ihren Zugangsdaten angemeldet haben, können Sie auf CME.SpringerMedizin.de die gewünschten CME-Kurse Ihrer Zeitschrift nutzen. Die Kurse können jederzeit unterbrochen und später fortgesetzt werden.
CME-Punkte sammeln

Zu jedem Fortbildungskurs gehört ein Fragebogen mit 10 CMEFragen. Mit 7 richtigen Antworten haben Sie bestanden und erhalten umgehend eine Teilnahmebescheinigung!

Teilnehmen und weitere Informationen unter:

CME.SpringerMedizin.de

Unser Tipp: Noch mehr Fortbildung bieten die e.Med-KombiAbos. Hier stehen Ihnen die CME-Kurse der Fachzeitschriften von Springer Medizin in elektronischer Form zur Verfügung. Auf Wunsch erhalten sie mit den e.Med-Kombi-Abos außerdem eine gedruckte Fachzeitschrift Ihrer Wahl.

Testen Sie e.Med 30 Tage kostenlos und unverbindlich!

Jetzt informieren unter

www.springermedizin.de/eMed

oder telefonisch unter 0800-77 80777

(Montag bis Freitag, 10 bis 17 Uhr) 